\title{
Efeito do exercício físico no envelhecimento: diferenças nas aptidões físicas entre idosos ativos e sedentários
}

Effect of physical exercise on aging: differences in physical fitness between active and sedentary elderly people

\section{Isabella Bernardes da Silva Medeiros \\ Centro Universitário Geraldo Di Biase - Fundação Educacional Rosemar Pimentel \\ isabellabernardes2@yahoo.com.br \\ Lucas Henrique Pessanha Ferreira}

Universidade Federal do Rio de Janeiro, Escola de Educação Física e Desportos, Departamento de Corridas

lucas.pessanha.1996@gmail.com

\section{Verônica Salerno}

Universidade Federal do Rio de Janeiro

vpsalerno@yahoo.com.br

\section{Diego Viana-Gomes}

Universidade Federal do Rio de Janeiro

diegoefd@gmail.com

Conflito de interesses: nada a declarar. Financiamento: nada a declarar.

Histórico:

Submissão | Received: 27/01/2022

Aprovação | Accepted: 31/01/2022

Publicação | Published: 01/02/2022

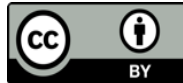

Todo o conteúdo do JIM - Jornal de Investigação Médica é licenciado sob Creative Commons, a menos que especificado de outra forma e em conteúdo recuperado de outras fontes bibliográficas. 


\section{RESUMO}

0 envelhecimento é um processo natural e irreversível, que causa a redução da autonomia e capacidade funcional. A prática regular de atividades físicas promove manutenção das capacidades funcionais do idoso. 0 objetivo desta pesquisa foi comparar a capacidade funcional e aptidão física, entre idosos ativos e sedentários. Foram avaliados 80 idosos com a idade média entre 60 e 80 anos, e o método de avaliação foi o Teste de Aptidão Física para Idosos (TAFI), que mensura a capacidade funcional do indivíduo através de testes de aptidão funcional e parâmetros físicos que estão relacionados às funções diárias. Através de todos os dados coletados pôde-se perceber uma diferença significativa na força muscular de membros superiores e inferiores, resistência aeróbica, flexibilidade de membros superiores, agilidade e equilíbrio dinâmico dos idosos ativos quando comparados aos idosos sedentários. Concluiu-se, a partir destes dados, que o exercício físico regular melhora a capacidade física dos idosos.

Palavras-Chave: Teste físico, envelhecimento, exercício físico, Sedentarismo, Inatividade

\section{ABSTRACT}

Aging is a natural and irreversible process that causes autonomy and functional capacity reduction. Regular physical exercise, besides curbing the sedentary lifestyle, promote maintenance of the functional capacities in elderly people. The aim of the present study was to compare the effect of exercise on functional abilities and physical fitness, comparing active and sedentary elderly. Eighty elderly people between 60 and 80 years old were evaluated with Physical Fitness Test for Elderly, this test measures the functional capacity of each person through functional fitness tests and physical parameters, on the other hand, are related to daily functions. Results showed a significant difference in upper and lower limb muscle strength, aerobic resistance, lower and upper limb flexibility, agility and dynamic balance, when compared active and sedentary elderly. It was concluded from these data that regular physical exercises improve and maintains functional capacity.

Keywords: exercise test, aging, frail elderly, exercise, physical inactivity 


\section{INTRODUÇÃO}

Com o aumento da perspetiva de vida, cresce a preocupação com a qualidade de vida e com o envelhecimento saudável (Argento, 2010). De acordo com a Pesquisa Nacional por Amostra de Domicílios Contínua (Pnad - 2016), divulgado pelo Instituto Brasileiro de Geografia e Estatística (IBGE, 2016) (Ministério da Sáude, 2016), afirma-se que entre 2012 e 2016 a população idosa (com 60 anos ou mais de idade) cresceu 16,0\%, chegando a 29,6 milhões de pessoas. 0 aumento do número de idosos está ligado ao aumento da expectativa de vida, que decorre do avanço tecnológico e científico, permitindo maior acesso ao conhecimento relacionado à saúde.

0 processo de envelhecimento ocorre de forma natural e irreversível, sendo um processo normal a qualquer ser humano. Esse processo conduz a uma redução da autonomia, capacidade funcional e o desgaste em vários sistemas, tais como: sistema cardiovascular, respiratório, muscular e ósseo. (Brito \& Litvoc, 2004). Essas alterações podem ocasionar limitações funcionais diárias, como: caminhar sozinho, vestir-se e fazer compras. Nesse sentido, o idoso perde autonomia, pois precisa de ajuda para executar tarefas do cotidiano. Em contraponto, o exercício físico é uma estratégia que pode ser utilizada para manter e melhorar a capacidade funcional.

Ao longo dos anos vêm sendo sugerido a prática de atividades físicas independentemente da idade para manter a vitalidade, melhorar a capacidade respiratória e cardiovascular, contribuindo para manutenção da autonomia à medida que se envelhece. Segundo a Organização Mundial da Saúde (OMS, 2018), a atividade física é definida como qualquer movimento corporal produzido pelo músculo esquelético que resulta num aumento do gasto energético. A prática regular de atividade física, além de coibir o sedentarismo, promove a manutenção das capacidades funcionais do idoso, contribui na melhoria das funções orgânicas (prevenindo perda da massa magra, óssea, muscular, força motora e da independência), cognitivas e principalmente no aperfeiçoamento de sua aptidão física. Atribuindo assim um avanço significativo na independência pessoal e na prevenção de doenças (Silva et al., 2012).

De acordo com a Organização Mundial da Saúde (2018), são consideradas pessoas ativas as que praticam atividades físicas moderadas com uma duração mínima de 30 minutos, somando 150 minutos por semana com intensidade moderada. E ao contrário do que muitos pensam, sedentários não são pessoas que não praticam atividade física, e sim pessoas que gastam poucas calorias (menos de $2.000 \mathrm{kcal} /$ semana) com atividades ocupacionais. Para medir a capacidade funcional de idosos, um dos testes mais utilizados é o Teste de Aptidão Física para Idosos (TAFI), através de testes de aptidão funcional e parâmetros físicos que, por sua vez, estão relacionados às funções diárias, necessidades pessoais, tarefas domésticas (Rikli \& Jones, 2008). 
Sendo assim, este artigo oriundo da pesquisa de campo, justifica-se pela necessidade de avaliar se há mudanças na capacidade funcional e aptidão física, comparando idosos ativos com idosos sedentários, com a finalidade de compreender qual a diferença da capacidade funcional entre pessoas ativas e sedentárias quando atingem a fase do envelhecimento, e como isso afeta diretamente na sua autonomia.

\section{METODOLOGIA}

Os dados foram coletados no município de Valença-RJ. Participaram da coleta 80 idosos com idade de 60 a 80 anos. Esses idosos foram divididos em dois grupos: Idosos Ativos e Idosos Sedentários ( $\mathrm{N}=40$ em cada grupo), cuja idade média apresentada foi de 64 ( \pm 5,20 anos) para o grupo de Idosos Ativos e de 70 ( \pm 5,86 anos) para Idosos Sedentários. Foi aplicado nos idosos o Teste de Aptidão Física para Idosos (TAFI), visando avaliar a diferença da aptidão física em Idosos Ativos (IA) e Idosos Sedentários (IS). Este teste consiste em sete estações simples, com o objetivo de avaliar a Força muscular de membros inferiores, Força muscular de membros superiores, Resistência Aeróbica, Flexibilidade de membros inferiores, Flexibilidade de membros superiores, Agilidade e equilíbrio dinâmico e Massa Corporal. A pesquisa contou com aprovação do Comitê de Ética em Pesquisa da Universidade Geraldo Di Biase (CAAE: 92962418.1.0000.5609) e para a participação na pesquisa foi solicitada a assinatura do Termo de Consentimento Livre e Esclarecido (TCLE) pelo participante.

\section{Avaliação Física:}

A aplicação do Teste de Aptidão Física para Idosos (TAFI), foi feito em uma única sessão para a coleta de dados sendo separados em Idosos Ativos (IA) e Idosos Sedentários (IS).

Teste de Levantar da Cadeira que tem por objetivo avaliar a força dos membros inferiores. 0 procedimento para a execução correta do teste deve ser feita da seguinte maneira: instruir o participante a sentar no meio do assento com as costas eretas, pés fixos no chão e braços cruzados na altura dos punhos contra o tórax. Ao comando de "Iniciar" o participante se levanta de forma completa e retorna à posição sentada. 0 escore corresponde ao total de movimentos de levantar e sentar realizados em 30 segundos. Esse teste é aplicado apenas uma vez.

Teste de Flexão de Cotovelo tem por objetivo avaliar a força dos membros superiores. Foi utilizado peso de 2,27 $\mathrm{Kg}$ para mulheres e 3,63Kg para homens. 0 procedimento para a execução correta do teste deve ser feito da seguinte maneira: pedir ao participante para sentar-se em uma cadeira com as costas eretas, com os pés fixos no chão e o lado dominante do corpo próximo á borda lateral do assento da cadeira. 0 braço cuja mão 
segura o peso deve ficar estendido ao longo do tronco, perpendicular ao chão. 0 participante deve segurar o peso com uma mão, partindo dessa posição o braço é flexionado em direção ao ombro, com a palma da mão gradativamente se voltando para cima durante a flexão. A mão juntamente com o peso retorna à posição completamente estendida na mesma posição inicial. 0 escore corresponde ao número total de flexões de cotovelo executadas em 30 segundos. Esse teste é aplicado apenas uma vez.

Teste de Marcha Estacionária de 2 (dois) minutos tem por objetivo avaliar a resistência aeróbica. 0 procedimento para a execução correta do teste deve ser feito da seguinte maneira: marcar com fita adesiva a altura de 76,2 centímetros na parede onde será executado o teste. Ao comando de "iniciar" o participante começa a marchar no lugar (sem correr), e de acordo com sua capacidade executar o movimento em um período de 2 minutos. Para a execução correta os dois joelhos devem levantar até a marca, porém só é contabilizado o número de vezes que o joelho direito atingir a marca. 0 escore corresponde ao número de passos realizados em 2 minutos, isto é, o número de vezes que a perna direita atinge a altura assinalada. Esse teste é aplicado apenas uma vez.

Teste de Sentar e Alcançar os Pés e tem por objetivo avaliar a flexibilidade dos membros inferiores (principalmente os músculos posteriores da coxa). 0 procedimento para a execução correta do teste deve ser feito da seguinte maneira: o participante deve-se sentar na beirada da cadeira, a dobra entre a superfície superior da coxa e a nádega deve coincidir com a beirada do assento da cadeira. Uma das pernas fica fletida com o pé fixo no chão. A outra perna é estendida o máximo possível na frente do quadril. 0 tornozelo é apoiado no chão, com o pé fletido a aproximadamente 90 graus. Com os braços para fora, as mãos sobrepostas e os dedos médios na mesma altura, o participante se curva lentamente para frente, flexionando a articulação do quadril para tentar alcançar ou ultrapassar os dedos do pé. 0 alcance máximo deve ser sustentado durante dois segundos. 0 participante deve praticar o teste em ambas as pernas para ver qual o melhor resultado. 0 escore corresponde à distância da ponta dos dedos até a ponta do tênis em centímetros. A ponta do tênis representa o ponto zero. Se as mãos não alcançarem esse ponto, registrar a distância como escore negativo (-); se os dedos médios tocarem os dedos do pé, registrar um escore de zero; e se passarem do ponto central, registrar a distância como escore positivo ( + ).

Teste de Alcançar as Costas e tem por objetivo analisar a flexibilidade dos membros superiores (ombro). 0 procedimento para a execução correta do teste deve ser feito da seguinte maneira: o participante deve ficar em pé e passar a mão selecionada sobre 0 mesmo ombro, com a palma e os dedos estendidos, tentando alcançar a parte central das costas. Observar que o cotovelo deve apontar para cima. Logo após pedir ao participante para colocar a outra mão em torno da parte posterior da cintura com a palma voltada para cima, tentando fazer com que os dedos de ambas as mãos se toquem ou se sobreponham. 0 escore é anotado após duas tentativas, levando-se em conta o número 
de centímetros mais próximos. Medir a distância da sobreposição ou a distância entre as pontas dos dedos médios, indicando o melhor escore. Atribuir escore negativo ( - ) se os dedos médios não se tocarem, escore zero se os dedos médios se tocarem ligeiramente e escore positivo $(+)$ se os dedos médios se sobrepuserem. Medir sempre a distância entre a ponta de um dos dedos e a ponta do outro, seja qual for o alinhamento nas costas.

Teste de Levantar e Caminhar tem por objetivo analisar a agilidade e o equilíbrio dinâmico. 0 procedimento para a execução correta do teste deve ser feito da seguinte maneira: colocar a cadeira contra a parede voltada diretamente para um cone colocado a distância de 2,5 metros medida a partir da parte traseira do cone até um ponto no chão situado no mesmo nível da parte da frente da cadeira. Instruir o participante para sentar na parte central do assento da cadeira com as costas eretas, os pés sobre o chão e as mãos sobre as coxas. Um pé deve ser colocado ligeiramente à frente do outro, com o tronco levemente inclinado para frente. Ao comando "iniciar" o participante deve se levantar da cadeira, caminhar o mais rápido possível até o cone, contorná-lo e voltar para a cadeira, sentando-se novamente. Acionar o cronômetro ao sinal "iniciar", independente se participante comece a se movimentar ou não, e pará-lo no exato instante em que a pessoa sentar na cadeira outra vez. 0 escore é registado após o participante executar duas vezes. Os dois tempos devem ser registados, assinalando o melhor.

\section{Avaliação Antropométrica:}

Avaliação de Altura e Peso e tem por objetivo avaliar o índice de assa corporal (IMC). 0 procedimento para a execução correta do teste deve ser feito da seguinte maneira: para a altura podemos fixar uma fita métrica ( 2 metros) na parede em sentido vertical com a extremidade zero ou chão. 0 participante deve ficar com a parte posterior da cabeça contra a parede (a parte central da cabeça deve ficar alinhada com a fita métrica) e os olhos voltados diretamente para frente. Colocar a régua (ou objeto semelhante) no topo da cabeça do participante e, sustentando-a no nível, encostá-la na fita métrica. A altura da pessoa corresponde ao escore em centímetros indicada na fita métrica.

Para a execução do peso, o participante deve remover peças de roupa que possam atrapalhar (jaquetas, casacos pesados, etc.). 0 participante deve subir na balança digital com os dois pés unidos e se manter naquela posição até a balança registar a massa corpórea $(\mathrm{Kg})$.

No escore é anotado a altura e o peso do participante na ficha de avaliação. 0 cálculo da massa corporal pode ser feito através do cálculo de IMC, onde divide-se o peso em quilogramas pela altura ao quadrado $\left(\mathrm{IMC}=\mathrm{Kg} / \mathrm{m}^{2}\right)$. 


\section{ESTATÍSTICA}

Para comparação estatística utilizou-se o software GraphPad Prism 6.0 (California, EUA). Verificou-se a distribuição das amostras pelo Shapiro-Wilk normality test, onde verificouse que os grupos apresentam distribuição não paramétrica. Por isso, utilizou-se o Mann Whitney test para comparação entre os grupos, considerando-se significativas as diferenças com $\mathrm{p}$ valor menor que 0.05 .

\section{RESULTADOS}

0 presente estudo contou com a participação de 80 idosos, que foram separados em dois grupos de Idosos Ativos e Idosos Sedentários com 40 idosos em cada grupo, no qual foram aplicados em ambos os grupos o Teste de Aptidão Física para Idosos (TAFI).

A Tabela 1 representa a média de idade dos Idosos Ativos e Sedentários e a média de peso, altura e IMC.

Tabela 1. Média de Idade, Peso, Altura e IMC em Idosos Ativos e Idosos Sedentários.

\begin{tabular}{ccc}
\hline & Idosos Ativos & Idosos Sedentários \\
\hline Idade & $64 \pm 5,20$ & $70 \pm 5,86$ \\
Peso & $72,8 \pm 14,98$ & $72,6 \pm 15,88$ \\
Altura & $1,59 \pm 0,07$ & $1,62 \pm 0,08$ \\
IMC & $28,96 \pm 5,51$ & $27,62 \pm 6,19$ \\
\hline
\end{tabular}

Com relação aos dados obtidos no "Teste de Levantar na Cadeira", podemos observar que de acordo com a média dos números de repetições do grupo de Idosos Ativos $(12,68$; $\pm 2,21)$ e Idosos Sedentários $(10,80 ; \pm 3,83)$ houve uma diferença de $17,4 \%$ no movimento de sentar e levar da cadeira, como podemos verificar na Figura 1.

De acordo com os dados obtidos no "Teste de Flexão de Cotovelo", podemos analisar que a média dos números de repetições do grupo de Idosos Ativos $(17,03 ; \pm 3,49)$ e Idosos Sedentários $(12,85 ; \pm 3,97)$ houve uma diferença de $32,5 \%$ no movimento de flexão de cotovelo, como podemos verificar na Figura 1. 
Figura 1. Avaliação da aptidão neuromuscular comparando os grupos de idosos sedentário $(n=40)$ e ativo $(n=40)$. A) Avaliação da força de membros inferiores através do teste de Levantar e sentar na Cadeira em 30 segundos, onde contabiliza-se o número de repetições; B) Avaliação da força de membros superiores através do teste de Flexão de Cotovelo unilateral com halter, onde foi avaliado o número de repetições em 30 segundos; ${ }^{*} p<0,05$.
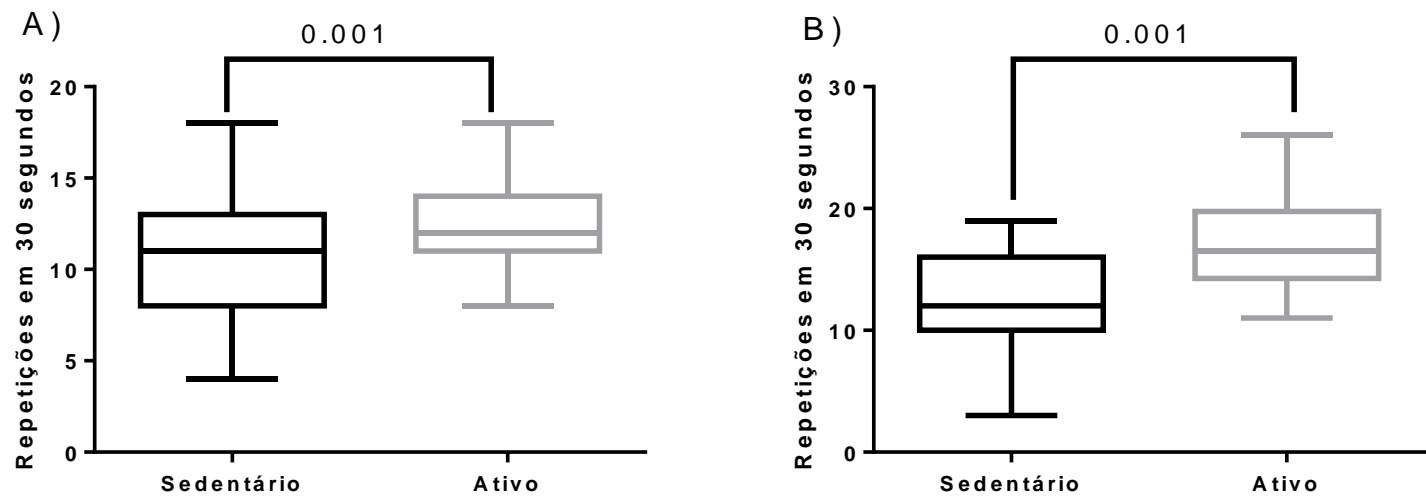

Ao analisarmos os dados obtidos no "Teste de Marcha Estacionária de 2min.", podemos verificar que a média do número de repetições do grupo de Idosos Ativos $(71,85 ; \pm 15,72)$ e Idosos Sedentários $(56.05 ; \pm 18,43$ ) houve uma diferença de $28,1 \%$ no movimento de caminhar, como podemos ver na Figura 2.

Figura 2. Avaliação da aptidão cardiorrespiratória comparando os idosos dos grupos sedentário $(n=40)$ e ativo $(n=40)$. ${ }^{*} p<0,05$.

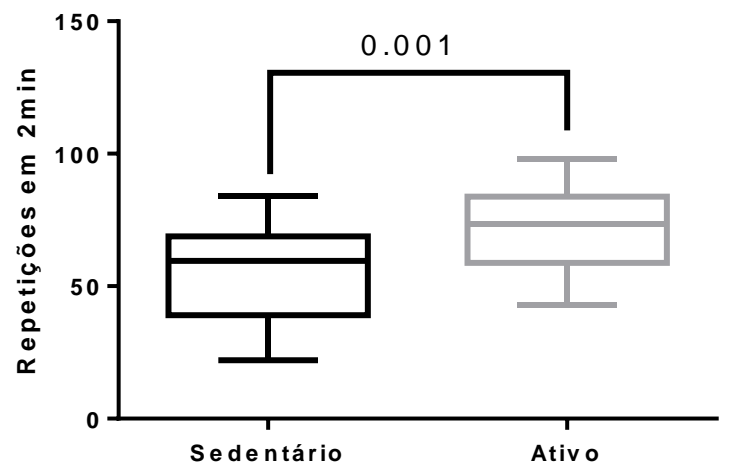

Ao examinar os dados obtidos no "Teste de Sentar e Alcançar os pés" verificou que a média dos centímetros do grupo de Idosos Ativos $(2,02 ; \pm 4,86)$ e Idosos Sedentários $(2,92 ; \pm 7,46)$ não houve uma diferença significativa no movimento de alcançar os pés, conforme podemos ver na Figura $3^{\text {a }}$. Com referência aos dados obtidos no "Teste de Alcançar as Costas" verificamos que a média dos centímetros do grupo de Idosos Ativos $(-6,4 ; \pm 6,5)$ e Idosos Sedentários $(-19,8 ; \pm 11,68)$ houve uma diferença de $209 \%$ no movimento de alcançar as costas, conforme observamos na Figura 3B. 
Figura 3. Avaliação da flexibilidade comparando os idosos dos grupos sedentário $(n=40)$ e ativo $(n=40)$. A) Teste de sentar e alcançar para avaliar a flexibilidade de membros inferiores; B) Teste de alcançar as mãos nas costas para avaliar a flexibilidade de membros superiores. ${ }^{*} \mathrm{p}<0,05$.

A)

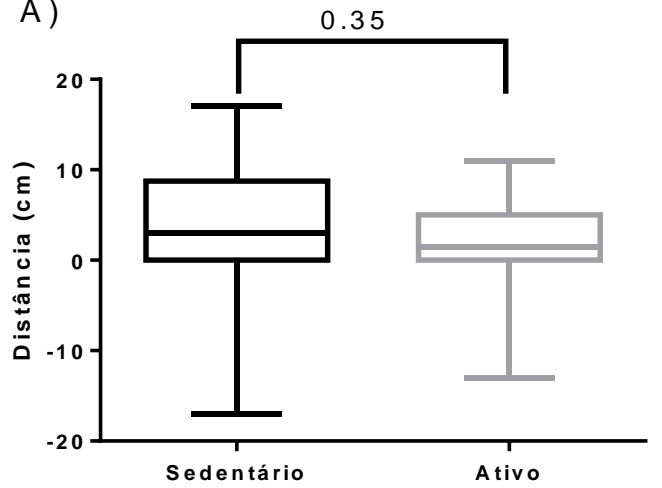

B)

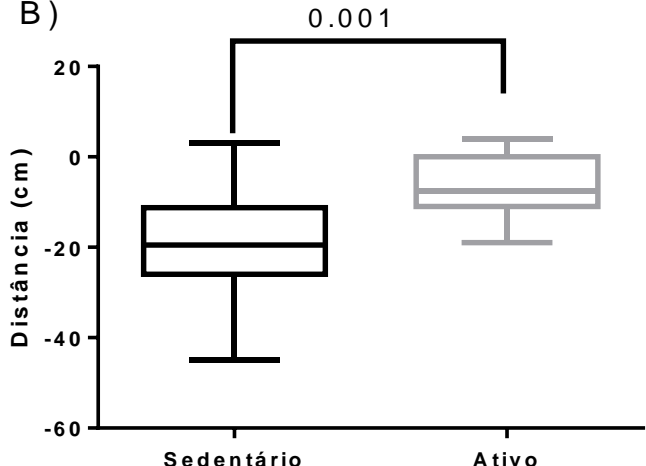

Nos dados obtidos no "Teste de Levantar e Caminhar" observou que a média de segundos do grupo de Idosos Ativos $(5,62 ; \pm 1,18)$ e Idosos Sedentários $(12,06 ; \pm 4,39)$ houve uma diferença de $114 \%$ no movimento de levantar e caminhar, conforme vemos na Figura 4.

Figura 4. Avaliação da aptidão neuromotora comparando os idosos dos grupos sedentário $(n=40)$ e ativo $(n=40)$. Teste de levantar, caminhar, voltar e sentar novamente (Time Up and Go) com o objetivo de analisar a agilidade e equilíbrio dinâmico. * ${ }^{*}<0,05$

\subsection{1}

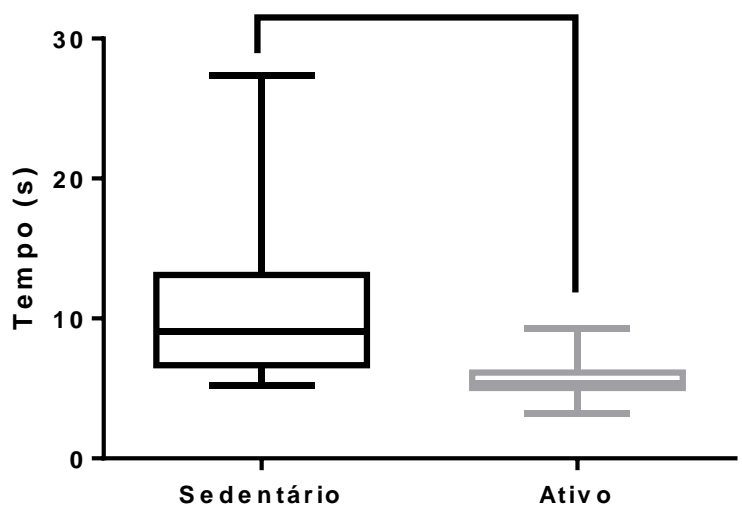

\section{DISCUSSÃo}

Através dos testes propostos, verificou-se que ocorreu mudanças nas capacidades físicas e funcionais entre idosos ativos e sedentários como: força, flexibilidade, agilidade, resistência aeróbica e equilíbrio dinâmico. Civinski, Montibeller \& Braz (2011) também corroboram com a ideia de que "a participação em um programa de exercícios físicos 
regulares é essencial, e uma forma efetiva para reduzir, prevenir e tratar declínios funcionais associados ao envelhecimento". Em outro estudo, pôde-se comprovar a influência do exercício físico na independência funcional e na qualidade de vida em idosos (Neto \& Castro, 2012).

Encontrou-se, nesta pesquisa, Índice de Massa Corpórea (IMC) parecidos entre ambos os grupos, as diferenças nos testes que medem as habilidades físicas apresentaram-se significativamente diferente. De acordo com a Figura $1 \mathrm{~A}$, pode-se verificar que houve uma diferença de $17,4 \%$ na força de membros inferiores para os Idosos Ativos. Essa diferença pode acarretar um grande desequilíbrio nas atividades diárias dos idosos e até mesmo ocasionar quedas. No estudo de Fhon et al. (2018), observou-se resultados que corroboram com os dados encontrados neste trabalho, apontando que houve diminuição da força de membros inferiores em idosos que não praticam exercícios físicos.

Ao analisar a Figura 1B, verifica-se uma diferença de $32,5 \%$ na força de membros superiores para os Idosos Ativos. A manutenção da força de membros superiores está relacionada com a autonomia para realizar atividades básicas diárias, e sua perda pode propiciar dificuldade para o idoso na execução dessas atividades, perda da autonomia e também acarretar a depressão, como descrito por Rodrigues et al. (2018).

Na Figura 2 houve melhora de 28,1\% na capacidade aeróbia dos Idosos Ativos, avaliada pelo teste de caminhar. Gonzaga et al., (2011) descreveram que não houve diferença significativa no teste de caminhada entre Idosos Ativos e Sedentários, sendo conflitante aos dados encontrados nessa pesquisa. A melhora da capacidade aeróbia permite que 0 idoso percorra maiores distâncias ou aumente a velocidade da caminhada sem que haja fadiga precoce. Com o envelhecimento a aptidão cardiorrespiratória tende a diminuir e 0 treinamento físico diminui a velocidade de perda e pode promover melhora, como mostrado nos idosos avaliados nesse trabalho.

Outra aptidão importante para autonomia das atividades diárias é a flexibilidade, pois indivíduos com pouca amplitude articular podem perder a capacidade de calçar sapatos, vestir as roupas e realizar sua higiene pessoal. A figura $3 \mathrm{~A}$ mostrou que a prática regular de exercício não melhorou a flexibilidade de membros inferiores. Este resultado pode estar relacionado à sarcopenia e limitações naturais do envelhecimento como, por exemplo, a redução de amplitude de membros inferiores, conforme afirma Souza \& Rodacki (2012). A figura 3B apresenta melhora significativa com diferença de $209 \%$ na flexibilidade de membros superiores dos Idosos Ativos. A flexibilidade de membros superiores está entrelaçada com diversos movimentos do cotidiano, proporcionando uma maior autonomia e qualidade de vida para esses idosos. Essa qualidade de vida e autonomia dos Idosos Ativos corrobora com um estudo de Silva et al., (2012) onde encontraram aumento da flexibilidade de Idosos praticantes de exercícios físicos. 
Além das aptidões descritas acima: neuromuscular, cardiorrespiratória e flexibilidade; fazse necessário avaliar a aptidão neuromotora, que está relacionada ao equilíbrio e coordenação motora, duas valências que sofrem perda significativa com 0 envelhecimento. Na Figura 4, constatou-se diferença de 114\% no movimento de levantar e caminhar dos Idosos Ativos, teste que permite estimar a capacidade de locomoção diária probabilidade de quedas. 0 grupo ativo demora menos tempo para levantar, caminhar, voltar e sentar do que o grupo sedentário, fato que também pode ser associado ao aumento das outras aptidões físicas. Todavia, esses resultados são distintos do estudo feito por Gonzaga et al. (2011), onde não encontraram diferença significativa na marcha em idosos praticantes de diferentes atividades semanais.

Os dados encontrados nesse estudo são semelhantes ao trabalho de Virtuoso et al. (2014), que usaram uma bateria de teste similar, corroborando com os dados de desempenho, mas mostraram que o exercício físico também melhora a qualidade de vida e autonomia em Idosos Ativos quando confrontado aos Idosos Sedentários. Como se já não bastassem os benefícios dos exercícios físicos regulares supracitados, Minghelli et al. (2013) também concluíram que "a prática de atividade física pode representar um fator importante para reduzir os níveis de ansiedade e depressão em idosos", ultrapassando os benefícios físicos/funcionais e atingindo também o emocional.

\section{CONCLUSÕES}

A partir dos resultados obtidos, através da comparação entre os dois grupos, foi possível observar melhores resultados nas aptidões físicas dos idosos praticantes de exercícios em comparação aos sedentários. Conclui-se que a prática regular de exercícios físicos influencia positivamente na vida dos idosos, melhorando a força, capacidade aeróbia, flexibilidade e aptidão neuromotora. Estes fatores podem contribuir no envelhecimento saudável e aumentar a autonomia de vida, mostrando assim a importância da prática regular de exercícios físicos planejados e acompanhados por um profissional de Educação Física. Estes fatores influenciam diretamente num envelhecimento saudável e consequente independência/autonomia diária do idoso. 


\section{BIBLIOGRAFIA}

Argento, R.S.V. (2010) Benefícios da Atividade Física na saúde e qualidade de vida do idoso. Trabalho de conclusão de curso (graduação). Bacharelado em Educação Física, Universidade Estadual de Campinas, Campinas, São Paulo, Brasil.

Brito, F.C. \& Litvoc, C. J. (2004) Envelhecimento - prevenção e promoção de saúde. São Paulo, SP, Brasil.

Civinski,C., Montibeller A., Braz, A.L.O. (2011) A importância do exercício físico no envelhecimento. Revista da Unifebe (Online). v.9, p.163-175, Jan/Jun.

Fhon, J.R.S. et al. (2018). Fatores associados à fragilidade em idosos: estudo longitudinal. Revista de Saúde Pública [online]. v. 52, 74.

Gonzaga, J.M. et al. (2011). Efeitos de Diferentes Tipos de Exercício nos Parâmetros do Andar de Idosas. Revista Brasileira Medicina Esporte. v 17, $n^{0} 3$.

IBGE. Disponível em: <https://agenciadenoticias.ibge.gov.br> Acesso em: 24 de Novembro de 2017.

Minghelli B., Tomé B., Nunes C., Neves A., Simões C. (2013). Comparação dos níveis de ansiedade e depressão entre idosos ativos e sedentários. Revista de Pesquisa Clínica. v. 40, p71-76.

Ministério da Sáude. Disponível em: <http://portalms.saude.gov.br> Acesso em: 01 de outubro de 2016.

Neto M.G., Castro M.F. (2012). Estudo Comparativo da Independência Funcional e Qualidade de Vida entre Idosos Ativos E Sedentários. Revista Brasileira Medicina Esporte. v.18, $n^{0} 4$.

OMS (2018). Plano De Ação Global Para A Atividade Física 2018-2030. https://apps.who.int/iris/bitstream/handle/10665/272721/WHO-NMH-PND-18.5por.pdf

Rikli R.E., Jones C.J. (2008) Teste de Aptidão Física para Idosos. $1^{\text {a }}$ ed. Barueri, SP: Manole, Brasil.

Rodrigues, R.A. P. et al. (2018) Síndrome da fragilidade entre idosos e fatores associados: comparação de dois municípios. Revista Latino Americana de Enfermagem. V. 26. 
Silva, M.F.D. et al. (2012). Relação entre os níveis de atividade física e qualidade de vida de idosos sedentários e fisicamente ativos. Revista Brasileira Geriatria e Gerontologia. v 15, p 635-642.

Souza R.M., Rodacki A.L. F. (2012). Análise da marcha no plano inclinado e declinado de adultas e idosas com diferentes volumes de atividades semanais. Revista Brasileira Medicina Esporte. v18, $n^{0} 4$.

Virtuoso, J.F. et al. (2014) Força de preensão manual e aptidões físicas: um estudo preditivo com idosos ativos. Revista Brasileira Geriatria e Gerontologia. v 17, p 775784. 